\title{
An Automated Coupled-Column Liquid Chromatography System for Measuring Aqueous Solubilities of Hydrophobic Solutes
}

\author{
John W. Owens and Howard DeVoe \\ University of Maryland, College Park, MD 20742 \\ and \\ Thomas J. Buckley and Stanley P. Wasik \\ National Bureau of Standards, Gaithersburg, MD 20899
}

\begin{abstract}
Accepted: October 11, 1984
An automated apparatus is described for measuring the aqueous solubility of a sparingly soluble organic compound at many different temperatures. Water is pumped through a generator column packed with a chromatographic support coated with the organic compound, producing a saturated solution. The solute in a measured volume of this solution is extracted with an extractor column and analyzed by high performance liquid chromatography (HPLC). The temperature of the thermostat bath and the operation of the valves and the HPLC are under the control of a microcomputer. Solubility measurements of ethylbenzene obtained with this apparatus have a standard deviation at any one temperature of about $3 \%$ of the mean.
\end{abstract}

Key words: automation; ethylbenzene; HPLC; hydrophobic; liquid chromatography; microcomputer; solubility; solute; water.

\section{Introduction}

Accurate measurements of the aqueous solubilities of hydrophobic substances as functions of temperature are needed for testing theoretical models of hydrophobic effects. Over the past few years a general method for measuring aqueous solubilities of solid and liquid hydrophobic compounds has been developed at the Center for Analytical Chemistry and the Center for Chemical
Physics at the National Bureau of Standards [1-4]'. In this method, which will be called coupled-column liquid chromatography (CCLC), a saturated solution of the solute is generated by pumping water through a thermostated generator column containing the solute coated on a chromatographic support. A measured volume of the solution passes through a small extractor column filled

${ }^{1}$ Figures in brackets indicate literature references.

About the Authors, Paper: John W. Owens worked on this project as a graduate student at the University of Maryland and an IPA (Intergovernmental Personnel Act) summer employee at NBS. Howard DeVoe, an associate professor in the Department of Chemistry at the University of Maryland and the author to whom correspondence should be addressed, was a guest worker and IPA summer employee at NBS. Thomas J. Buckley and Stanley P. Wasik are research chemists in the NBS Center for Chemical Physics. The work presented here is taken from a thesis prepared by Mr. Owe___ and those of Professor DeVoe were supported in part by partial fulfillment of the M.S. degree. 
with a superficially-porous reverse phase packing where the solute is removed quantitatively. The extracted solute is eluted from the extractor column with a waterorganic mobile phase onto a reverse phase analytical column where the solute is separated from impurities and its amount determined by high performance liquid chromatography (HPLC) with ultraviolet detection. The method minimizes or avoids experimental problems inherent in the usual "shake-flask" method (formation of colloidal dispersions, errors from adsorption on walls, and loss of volatile solutes to the atmosphere), and also has advantages of speed and ease of thermostating [3].

This paper describes our exploitation of an additional advantage of the CCLC method, i.e., the ease with which the solubility measurements can be automated. We have assembled an automated CCLC system in which manual control of the required sequence of valve switchings, HPLC pump settings, and integrator functions is replaced with timed control signals from a microcomputer in order to eliminate operator error. Because the automated system can run unattended overnight, the productivity of the laboratory is greatly increased. An important additional feature is automatic temperature control of the generator column, so that the solubility of a solute at many pre-selected temperatures can be measured for subsequent calculation of the enthalpy of solution through the van't Hoff method. This paper describes the construction and operation of the system and evaluation of some results obtained for ethylbenzene, a liquid solute.

\section{Operation of the CCLC System}

This section describes general aspects of the operation of the CCLC system that are common to both manual and automated control. Details of individual components of the system are given in section 3 .

Figure 1 is a block diagram of the entire system. Water is pumped through the generator column from the generator column pump at a constant flow rate, typically $0.3 \mathrm{~mL} / \mathrm{min}$. Because of the large interfacial area and small diffusion path in the generator column, the water rapidly becomes saturated with the solute. Internal surfaces between the generator column and Valve 1 become equilibrated with the saturated solution before measurements are made, so that no further adsorption occurs here that would introduce errors by removing solute from solution.

The HPLC pump supplies a mobile phase composed of water, methanol, or a water-methanol mixture. The composition of the mobile phase and the flow rate are
Valve 1 is a two-position switching valve. In the Extract position, with interport connections shown as heavy solid lines in figure 1 , the saturated aqueous solution from the generator column passes through the extractor column and out to waste (labeled W in fig. 1) and the mobile phase from the HPLC pump continues on to Valve 2. In the Analyze position of Valve 1 (heavy broken lines) the aqueous solution goes directly to waste and the mobile phase passes through the extractor column and then continues on to Valve 2 . Valve 2 is a two-position switching valve that allows the mobile phase to either pass through the analytical column (connections shown by heavy solid lines) or to bypass the analytical column (heavy broken lines). The valve positions are changed by actuators that can be operated either manually (using electric switches) or by commands from the microcomputer. The generator and extractor columns, Valve 1, and interconnecting tubing are thermostated in a water bath (indicated by the dashdot rectangle in fig. 1) whose temperature is monitored with a quartz crystal thermometer (QCT) and controlled with two heating elements $(\mathrm{H})$.

The signal peak from the ultraviolet detector (D) is integrated by the integrator (I).

The sequence of operations for a typical run (one solubility measurement) is shown in figure 2 . While these tedious operations could be carried out manually, automated control has the distinct advantage of eliminating procedural errors. The four main steps of the run, labeled Condition, Extract, Analyze, and Strip, are as follows:

1. Condition. In this step pure water from the HPLC pump displaces methanol in the extractor column in order to condition this column for extraction. Valve 2 is placed in the Bypass position to prevent pure water from entering the analytical column which contains pure methanol from the initial setup or the preceding run. (We have found that it is advantageous to avoid in this way a sudden drastic change of the mobile phase composition in the analytical column, since such changes tend in time to damage the column packing as shown by broadened peak widths.) During this step the saturated aqueous solution from the generator column is diverted to waste.

2. Extract. Valve 1 is placed in the Extract position, switching the saturated aqueous solution from the generator column into the extractor column where the solute is extracted. The water remaining from extraction goes to waste. The mobile phase is changed to a composition ( $80 \%$ methanol in this example) that will later give a convenient retention time for the solute peak during HPLC analysis. After a brief delay to allow the new composition to reach Valve 2, Valve 2 is switched 


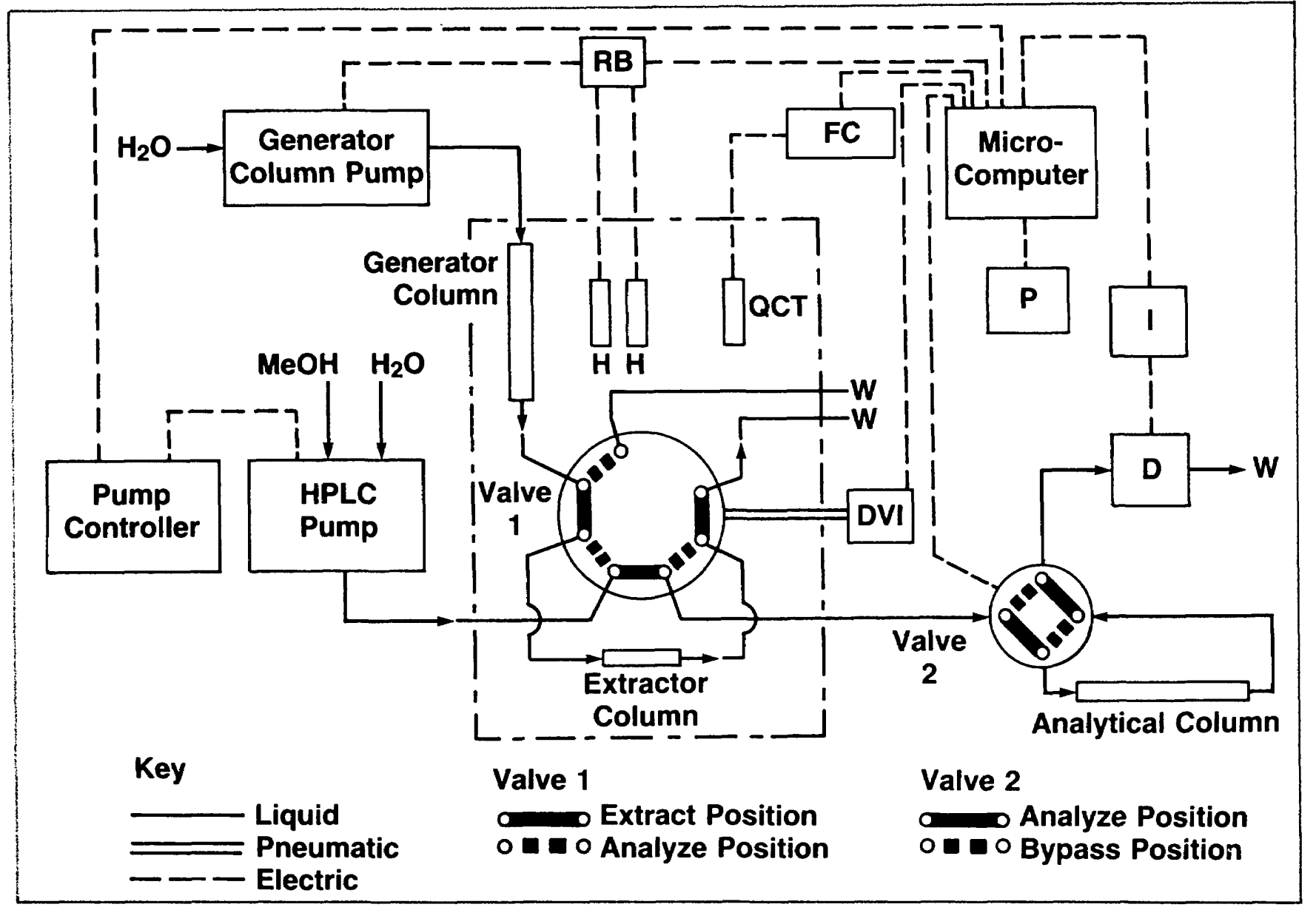

Figure 1-Block diagram of automated CCLC system. Abbreviations: D, detector; DVI, digital valve interface; FC, frequency counter; H, heater; I, integrator; $\mathrm{MeOH}$, methanol; P, printer; QCT, quartz crystal thermometer; RB, relay box; W, waste. The components shown within the dash-dot rectangle are thermostated.



Figure 2-Timing diagram for a typical solubility run. In this example the solute is ethylbenzene and the flow rate through the generator column is $0.3 \mathrm{~mL} / \mathrm{min}$. Time along the horizontal axis is measured from the beginning of the Condition step. 
to the Analyze position and the mobile phase enters the analytical column. The flow rate of the mobile phase is reduced to $0.1 \mathrm{~mL} / \mathrm{min}$ to conserve solvents, and later it is returned to the value needed for HPLC analysis (2 $\mathrm{mL} / \mathrm{min}$ in the example shown in fig. 2). (The microcomputer program calculates the duration of the period of reduced flow rate so as to condition the analytical column with a predetermined volumn of mobile phase. This period is longer for runs with less-soluble solutes requiring longer extraction times.) The Extract step is precisely timed for later calculations.

3. Analyze. Valve 1 is changed to the Analyze position and the integrator is started. The methanol-water mobile phase elutes the solute, extracted during the preceding Extract step, from the extractor column and focuses it on the head of the analytical column where chromatographic analysis begins. The eluted solute includes the total amount that was present in the extracted aqueous solution, provided that there was no breakthrough of solute from the extractor column during the preceding Extract step. The integrator plots a chromatogram during the analysis. After the solute peak has been integrated, the integration is stopped and the integrator prints the peak area on the chromatogram chart.

4. Strip. Pure methanol is pumped from the HPLC pump through the extractor and analytical columns to strip off any organic impurities that were retained by these columns.

After the strip step, there is a choice of operations which in the case of automated control is preprogramed by the operator. The choices are three: a new run at the same temperature can be started; the bath temperature can be changed and the next run begun after a time delay for re-equilibration of the generator column at the new temperature; or the measurements can be ended.

\section{Components and Interfaces}

The columns, pumps, valves, and detector are connected by $1 / 16$-in $(1.6 \mathrm{~mm})$ OD stainless steel (ss) tubing and standard tube connectors. The appendix lists the sources of certain of the components used in the CCLC system.

\subsection{Columns}

Figure 3 shows the design of a generator column that is suitable for generating saturated aqueous solutions of an organic liquid. The column is constructed from stainless steel for strength.

The body of the column consists of a $25-\mathrm{cm}$ length of $1 / 4$-in $(0.64 \mathrm{~cm})$ o.d. ss tubing connected to a $3.8-\mathrm{cm}$ length of $3 / 8$-in $(0.95 \mathrm{~cm})$ o.d. ss tubing. These two sections were joined by inserting one end of the $3 / 8$-inch

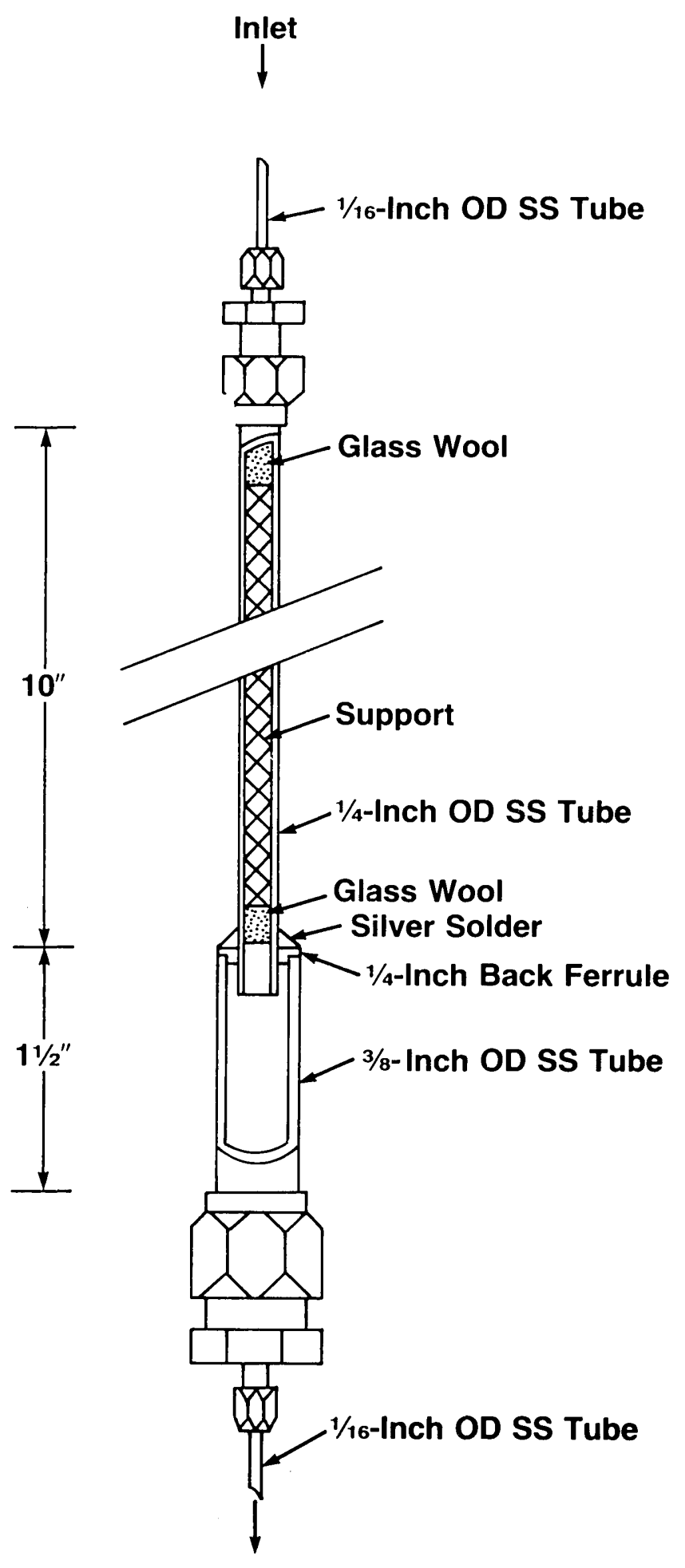

\section{Outlet}

Figure 3-Stainless steel generator column for producing a saturated aqueous solution of a liquid solute. See text for details. 
tube over the smaller-diameter end of a ss back ferrule designed for 1/4-in tubing; placing one end of the 1/4-in tube through the back ferrule; and silver soldering the three pieces together. The column has ss reducing end fittings for 1/16-in ss tubing, and is packed with a silanized diatomaceous silica support that is retained in the smaller-diameter section of the body by two plugs of glass wool.

To load the generator column with an organic solute for a series of runs, the column is mounted vertically (as shown in fig. 3) and about $1 \mathrm{~mL}$ of solute is pulled downward through the packing using gentle suction. The column is then filled slowly from the bottom with water so as to avoid trapping air. During runs the column remains in this vertical position but the flow of water is downwards. The short section of largerdiameter tubing at the bottom of the column serves as a trap for droplets of the organic liquid pushed off the packing, preventing them from reaching the outlet tube. The organic liquid, being less dense than the aqueous solution, stays at the top of the trap; this behavior has been observed in a glass version of the generator column [3].

The extractor column is a $5.1 \mathrm{~cm} \times 0.6 \mathrm{~cm}$ ss tube with ss end fittings containing $5-\mu \mathrm{m}$ frits, filled with a superficially porous reverse phase packing. The analytical column is a commercial $30 \mathrm{~cm} \times 0.6 \mathrm{~cm}$ ss column containing a $10-\mu \mathrm{m}$ totally porous reverse phase $C_{18}$ packing.

\subsection{Pumps}

Water (HPLC grade) is delivered to the generator column with a solvent metering pump capable of pumping at pressures up to $34 \mathrm{MPa}(5,000 \mathrm{psi})$. The flow rate can be selected in increments of $0.1 \mathrm{~mL} / \mathrm{min}$.

The HPLC pump mixes two solvents in any desired proportion, pressurizes the mixture, and delivers it at pressures up to $51 \mathrm{MPa}$ with any desired flow rate between 0.06 and $10.23 \mathrm{~mL} / \mathrm{min}$. HPLC-grade water and methanol are the solvents in our measurements. They are degassed before use by rapidly bubbling helium through them in their separate containers for 10 minutes, and kept degassed during runs with a continuous reduced flow of helium. The mobile phase composition and flow rate are controlled by a pump controller connected electronically with the HPLC pump. This pump controller is interfaced to the microcomputer by an RS232 serial port provided on the controller for remote control.

\subsection{Valves}

Valve 1 is a 10-port high pressure switching valve turned by an air actuator operated with pneumatic pulses from a digital valve interface. (Figure 1 shows only the seven functional ports of Valve 1.) Valve 2 is a 4-port high pressure switching valve turned by an electric actuator. Both valves are built of 300 series stainless steel and Valcon S fluorocarbon, and are designed for a temperature range of $-198^{\circ} \mathrm{C}$ to $175^{\circ} \mathrm{C}$ and for pressures up to $48 \mathrm{MPa}$. The microcomputer controls the positions of both valves with TTL logic pulses.

\subsection{Detector and Integrator}

The absorbance signal from the ultraviolet detector (wavelength $254 \mathrm{~nm}$ ) goes to a recording integrator equipped with a digital input port for remote starting and stopping of integration. The integrator automatically prints peak areas after integration is stopped.

\subsection{Temperature Control}

Valve 1 and the extractor column are immersed in a 5-liter water bath stirred with a stirrer-circulator. The generator column, mounted over the bath in a vertical position, is enclosed in a glass jacket through which water from the bath is circulated. The bottom of the jacket dips into the bath so that the generator column and the tubing connecting its lower end to Valve 1 are at the same temperature as the bath. The water entering the top of the generator column is brought to the temperature of the bath in a length of stainless steel tubing coiled inside the jacket.

The bath is heated by two independent heating elements and cooled by refrigerated water circulated through a cooling coil. The microcomputer controls the bath temperature with TTL logic pulses that switch solid-state relays to turn the heating elements on and off. One heating element is used to regulate the bath temperature while both are used to raise the temperature to a higher setting.

The microcomputer monitors the bath temperature during runs by reading the frequency of a quartz crystal oscillator thermometer immersed in the bath. The oscillator frequency was calibrated as a function of temperature.

\subsection{Microcomputer}

A microcomputer with monitor, disk drive, and printer is used for automated control of the system.

A data acquisition card added to the microcomputer provides a timer and two digital 8-bit (parallel) input/ output ports. The timer is used to count the time intervals between control signals. Six of the bits from one digital output port are used to control the positions of Valves 1 and 2, the integrator start/stop function, and relays for power to the generator column pump and the two bath heater elements. 
A multifunction card added to the microcomputer provides a real-time clock, a parallel port and a serial port. The parallel port is connected to a printer. The serial port is used to control the HPLC pump parameters (mobile phase composition and flow rate) with serial commands to the pump controller.

A custom made interface card enables the microcomputer to read the nine binary coded decimal digits of the frequency counter two at a time.

The microcomputer control program for the CCLC system is written in BASIC. Flowcharts of the program are given in figure 4. After loading the generator column with solute and installing it in the system, the operator starts the program and enters appropriate parameter values into the microcomputer. These parameters include the desired temperatures and number of runs at each temperature, the time delays needed for reequilibration of the generator column after each temperature change, the mobile phase composition and flow rate for analysis, and the time intervals of the various steps for each run. The runs are then performed under microcomputer control as shown in figure 4(a).

Each interval between control signals for the pumps, valves and integrator is timed by the subroutine shown in figure 4(b). During the interval, the subroutine continuously reads the frequency of the quartz crystal thermometer, computes the bath temperature to an accuracy of $0.002{ }^{\circ} \mathrm{C}$ from a linear function of the frequency, and turns a bath heater element on or off to regulate the temperature. The subroutine also updates a monitor display of the bath temperature and the current status of the CCLC system. At the beginning and end of each Extract step, the printer provides permanent hard copy of the clock time (from the multifunction card) and bath temperature. From this information the duration of the Extract step can be calculated to the nearest second.

After all runs for a solute are completed, the microcomputer turns the pumps and bath heaters off. The monitor displays the time at which the experiment ended.

\section{Results}

The flow rate of the generator column pump was measured by weighing the water delivered during timed intervals, and was found to have a reproducibility at the lowest pump settings of $\pm 0.001 \mathrm{~mL} / \mathrm{min}$. The microcomputer regulated the bath temperature, as monitored

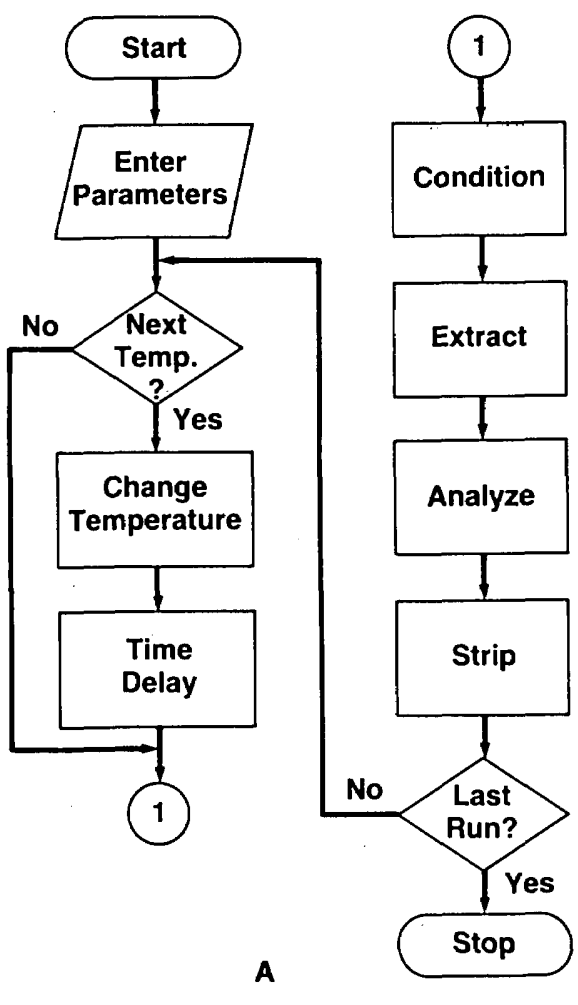

Figure 4-Flowcharts of the control program: A, left, the logic for a series of runs with one solute at one or more temperatures, and $B$, the subroutine for timing an interval between control signals for the pumps, valves, and integrator.

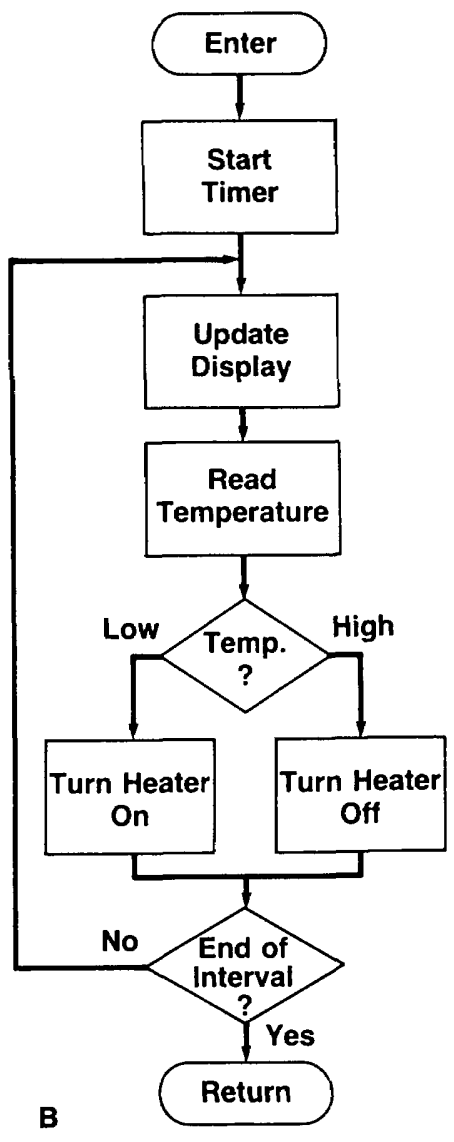


by the quartz crystal thermometer, to within $\pm 0.025^{\circ} \mathrm{C}$ of the set point at all temperatures.

The system described here has been used with the generator column shown in figure 3 for measurements of the aqueous solubilities of five liquid $n$-alkylbenzenes in the range $10-45^{\circ} \mathrm{C}$. The solubility, $s$, was calculated from each run using the relation

$$
s=n / V
$$

where

$$
\begin{aligned}
n & =\text { moles of solute } \\
& =\text { measured peak area } \times \text { detector response factor } \\
V & =\text { solution volume } \\
& =\text { extract step duration time } \times \text { generator column } \\
& \text { flow rate }
\end{aligned}
$$

The detector response factor for each solute was evaluated by injections of a standard methanol solution of the solute into the analytical column from a sample loop, the loop volume having been determined by a spectroscopic method [3].

Figure 5 shows data obtained for ethylbenzene. There is a total of 87 solubility values at 16 temperatures in the range $10-45^{\circ} \mathrm{C}$. The standard deviation of solubilities measured at any one temperature ranges from $0.006 \mathrm{mM}$ to $0.10 \mathrm{mM}$, with an average standard deviation for all temperatures of $0.05 \mathrm{mM}$ ( $3 \%$ of the mean). The points are scattered, but their large number allows the de- pendence of solubility on temperature to be determined statistically with reasonable certainty. A solubility minimum is evident at $20-24{ }^{\circ} \mathrm{C}$; Bohen and Claussen [5] observed such a minimum for ethylbenzene at about 18 ${ }^{\circ} \mathrm{C}$. The solubility and $95 \%$ confidence limit at $25{ }^{\circ} \mathrm{C}$ were calculated by the method of Clarke and Glew [6] to be $(1.74 \pm 0.02) \mathrm{mM}$, in good agreement with the value $(1.76 \pm 0.02) \mathrm{mM}$ obtained by Tewari et al. [7] at this temperature with the manual CCLC method. Other methods have yielded solubility values for $25^{\circ} \mathrm{C}$ ranging from $1.23 \mathrm{mM}$ to $2.00 \mathrm{mM}$ [5, 8-16]. The detailed results of our measurements on $n$-alkylbenzenes will be submitted for publication elsewhere.

\section{Conclusions}

The automated CCLC system described in this paper for measuring aqueous solubilities has proved to be convenient to use and considerably reduces the time needed to obtain reliable solubility values at many different temperatures.

The generator column described in this paper was built by Albert Ledford of NBS, and the oscillator frequency of the quartz crystal oscillator thermometer that was used in the work was calibrated by David Ditmars, also of NBS.

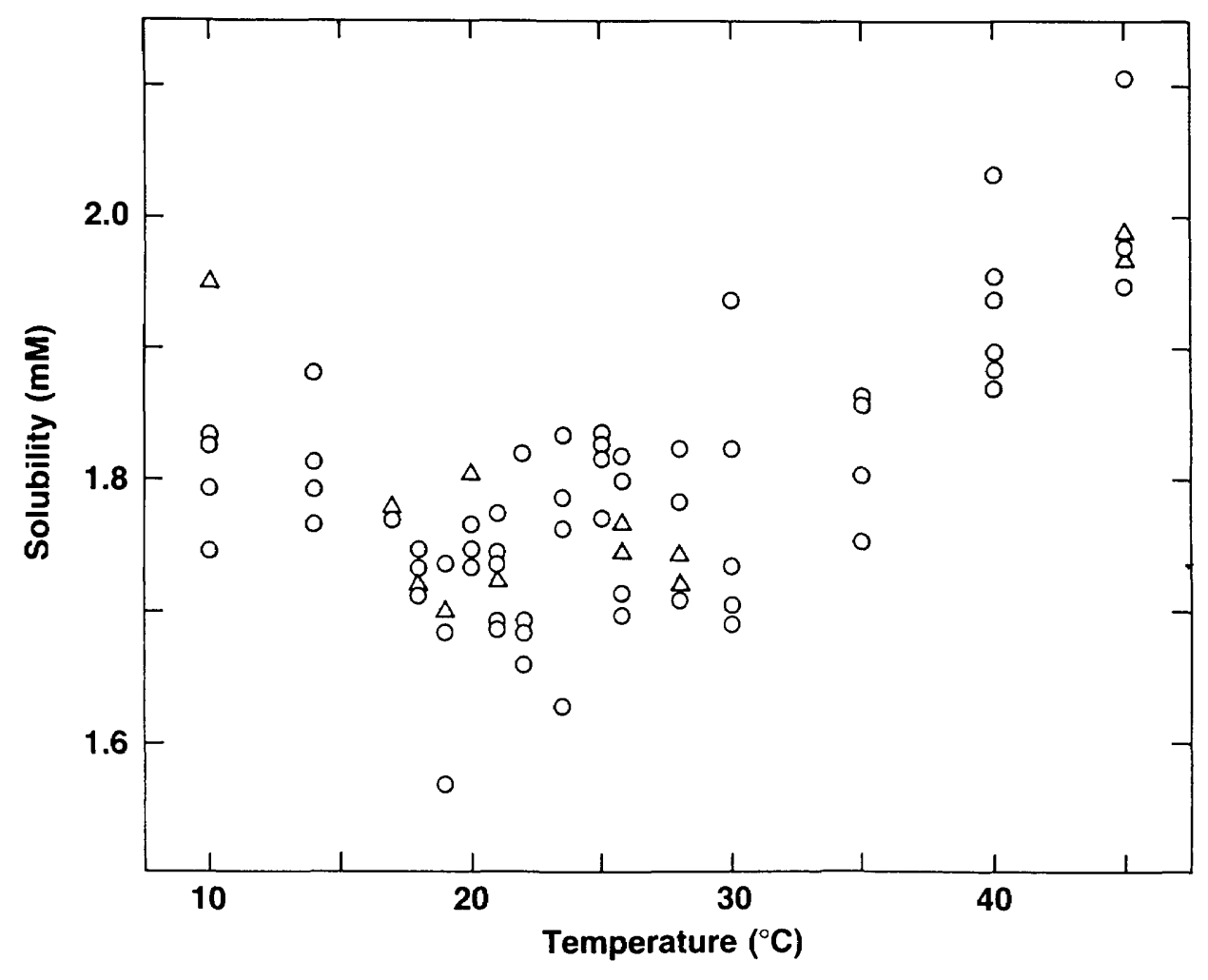

Figure 5-Measurements of the aqueous solubility of ethylbenzene at different temperatures. Each circle shows the result of an individual run. Sometimes two or more runs at the same temperature gave solubility values too close together ( $0.005 \mathrm{mM}$ or less) to be drawn as individual points; in these instances, the average of the values is shown as a triangle. 


\section{Appendix: Sources of Components (partial list)}

Certain trade names and company products are identified in order to adequately specify the apparatus and experimental procedure. In no case does such identification imply recommendation or endorsement by the National Bureau of Standards, nor does it imply that the products are necessarily the best available for the purpose.

Generator column packing-Chromosorb W, 100-120 mesh, high performance grade, Johns-Manville.

Extractor column packing-Bondapak $\mathrm{C}_{18} /$ Corasil, Waters Associates.

Generator column pump-Beckman model 110A.

HPLC pump-DuPont 8800 series analytical binary pump module.

Pump controller-DuPont 8800 series binary solvent controller.

Valve 1-Valco model AC10U valve and air actuator with 6 -in standoff.

Valve 2-Valco model EC4U valve and electric actuator.

Digital valve interface-Valco DVI digital interface.

Quartz crystal thermometer-Hewlett-Packard model $2830 \mathrm{~A}$.

Integrator-Hewlett-Packard model 3390A recording integrator with 19402A remote start/stop board.

Microcomputer-Apple II +.

Microcomputer cards-ADALAB data acquisition card, Interactive Microwave, Inc.; CPS multifunction card, Mountain Computer, Inc.

\section{References}

[1] May, W. E.; S. P. Wasik and D. H. Freeman. Determination of the aqueous solubility of polynuclear aromatic hydrocarbons by a coupled column liquid chromatographic technique, Anal. Chem. 50: 175-179 (1978).

[2] May, W. E.; S. P. Wasik and D. H. Freeman. Determination of the solubility behavior of some polycyclic aromatic hydrocarbons in water, Anal. Chem. 50: 997-1000 (1978).

[3] DeVoe, H.; M. M. Miller and S. P. Wasik. Generator columns and high pressure liquid chromatography for determining aqueous solubilities and octanol-water partition coefficients of hydrophobic substances, J. Res. Natl. Bur. Stand. 86: 361-366 (1981).

[4] Wasik, S. P.; M. M. Miller, Y. B. Tewari, W. E. May, W. J. Sonnefeld, H. DeVoe and W. H. Zoller. Determination of the vapor pressure, aqueous solubility, and octanol/water partition coefficient of hydrophobic substances by coupled generator column/liquid chromatographic methods, Residue Reviews 85: 29-42 (1983).

[5] Bohen, R. L., and W. F. Claussen. The solubility of aromatic hydrocarbons in water, J. Am. Chem. Soc. 73: 1571-1578 (1951).

[6] Clarke, E. C. W., and D. N. Glew. Evaluation of thermodynamic functions from equilibrium constants, Trans. Faraday Soc. 62: 539-547 (1966).

[7] Tewari, Y. B.; M. M. Miller, S. P. Wasik, and D. E. Martire. Aqueous solubility and octanol/water partition coefficient of organic compounds at $25.0^{\circ} \mathrm{C}, \mathrm{J}$. Chem. Eng. Data 27: 451-454 (1982).

[8] Andrews, L. J., and R. M. Keefer. Further studies on the argentation of substituted benzenes, J. Am. Chem. Soc. 72: 5034-5037 (1950).

[9] Klevens, H. B. Solubilization of polycyclic hydrocarbons, J. Phys. Colloid Chem. 54: 283-298 (1950).

[10] Morrison, T. J., and F. Billet. The salting-out of nonelectrolytes. II. The effect of variation in non-electrolyte, J. Chem. Soc. 3819-3822 (1952).

[11] McAuliffe, C. Solubility in water of paraffin, cycloparaffin, olefin, acetylene, cycloolefin, and aromatic hydrocarbons, J. Phys. Chem. 70: 1267-1275 (1966).

[12] Polak, J., and B. C. Lu. Mutual solubilities of hydrocarbons and water at 0 and $25^{\circ} \mathrm{C}$, Can J. Chem. 51: 4018-4023 (1973).

[13] Sutton, C., and J. A. Calder. Solubility of alkylbenzenes indistilled water and seawater at $25.0^{\circ} \mathrm{C}, \mathrm{J}$. Chem. Eng. Data 20: 320-327 (1975).

[14] Price, L. C. Aqueous solubility of petroleum as applied to its origin and primary migration, Bull. Am. Soc. Assoc. Pet. Geol. 60: 213-244 (1976).

[15] Ben-Naim, A. and J. Wilf. Solubilities and hydrophobic interactions in aqueous solutions of monalkylbenzene molecules, J. Phys. Chem. 84: 583-586 (1980).

[16] Sanemasa, I.; M. Araki, T. Deguchi, and H. Nagai. Solubility measurements of benzene and the alkylbenzenes in water by making use of solute vapor, Bull. Chem. Soc. Japan 55: 1054-1062 (1982). 